\title{
EXPERIMENTAL STUDY AND PERFORMANCE OF STRENGTH PROPERTIES OF CALCINED KAOLIN AND SILICA FUME PARENTAGE ADDITION
}

\author{
Kamar Elahi ${ }^{1} \boldsymbol{\bigotimes}$, Prof. Harsh Gupta ${ }^{2}$ \\ ${ }^{1}$ Research scholar, Department of Civil Engineering, Jawaharlal Nehru College of Technology, \\ Rewa (M.P.), India \\ 2 Professor, Department of Civil Engineering, Jawaharlal Nehru College of Technology, Rewa \\ (M.P.), India
}

DOI: https://doi.org/10.29121/granthaalayah.v9.i1.2021.3161

Article Type: Research Article

Article Citation: Kamar Elahi, and

Prof. Harsh Gupta. (2021).

EXPERIMENTAL STUDY AND

PERFORMANCE OF STRENGTH

PROPERTIES OF CALCINED KAOLIN

AND SILICA FUME PARENTAGE

ADDITION. International Journal of

Research -GRANTHAALAYAH, 9(1),

216-221.

https://doi.org/10.29121/granthaa

layah.v9.i1.2021.3161

Received Date: 05 January 2021

Accepted Date: 31 January 2021

Keywords:

Silica Fume

Compressive Strength

Tensile Strength

Sand

\begin{abstract}
The article is presenting the various type of strength like as compressive and tensile strength during the time of 7 and 28 days. The specimen ore making as $15 \mathrm{~cm} \mathrm{X} 15 \mathrm{~cm} \mathrm{X} 15 \mathrm{~cm}$ size for the testing purpose which is depending on the aggregate size. The results are show that strength performances of concrete with durability aspect are improved using Silica fume parentage addition.
\end{abstract}

\section{INTRODUCTION}

We are study about the property of test specimen which are having size of $15 \mathrm{~cm} \mathrm{X} 15 \mathrm{~cm} \mathrm{X} 15 \mathrm{~cm}$. The property of composition is generally used such as compressive strength and tensile strength. We are tested Mix (M1, M2, M3, M4, M5, M6, M7 and M8) to observe the suitable mix for construction propose, which is making with the help of Calcined Kaolin, Sand, Aggregate and composition.

\section{COMPRESSIVE STRENGTH FORMULA}

Compressive Strength $=$ Load $/$ Cross-sectional Area

(C) 2021 The Author(s). This is an open access article distributed under the terms of the Creative Commons Attribution License, which permits unrestricted use, distribution, and reproduction in any medium, provided the original author and source are credited. 


\section{PROCEDURE}

First of all, we are constructed the cube with size of $15 \mathrm{~cm} \mathrm{X} 15 \mathrm{~cm} \mathrm{X} 15 \mathrm{~cm}$ for testing propose and observing using concrete is completely poured and mould with temperature for the propose of removing the voids after one day cubical mould are removed and the specimen are placed in water tank for the propose of curing. All specimens after time of 7 and 28 days curing, we are tested with testing machine. The load id applied on the specimen $200 \mathrm{Kg} /$ $\mathrm{cm}^{2}$ per minutes gradually upto fails.

\section{PREPARATIONS}

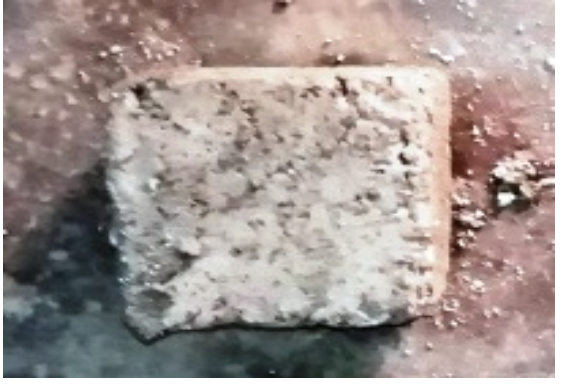

Figure 1: First Specimen

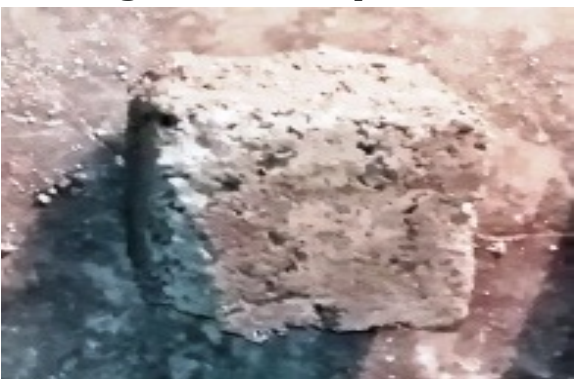

Figure 3: Third Specimen

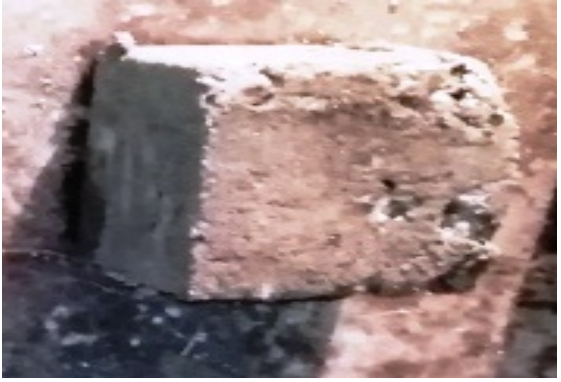

Figure 5: Fifth Specimen

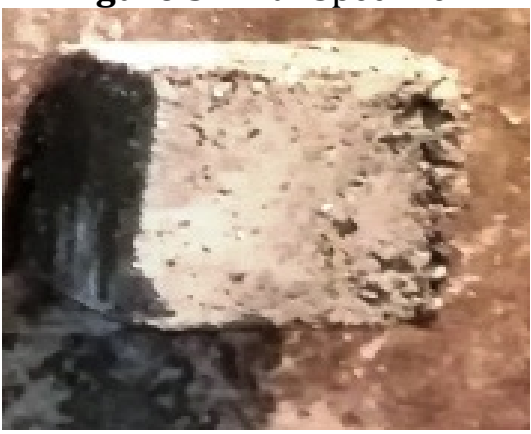

Figure 7: Seventh Specimen

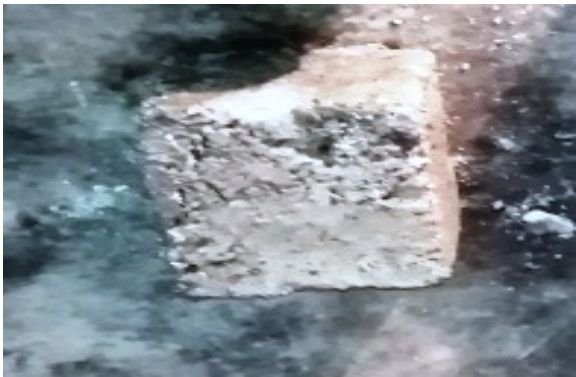

Figure 2: Second Specimen

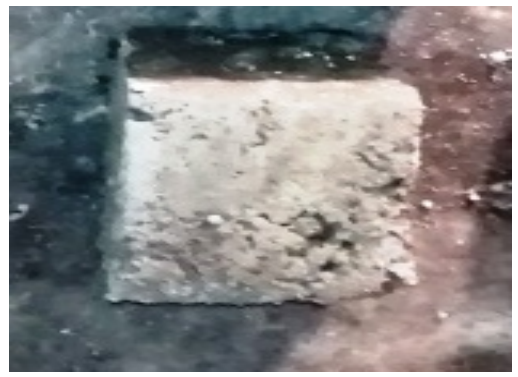

Figure 4: Fourth Specimen

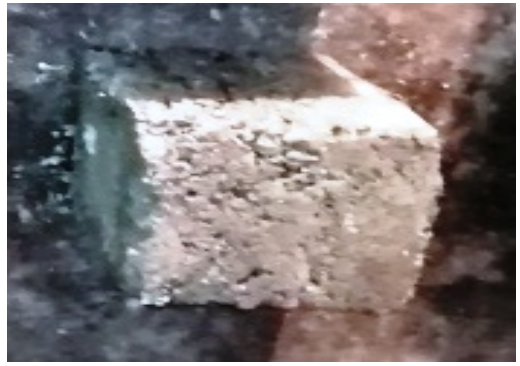

Figure 6: Sixth Specimen

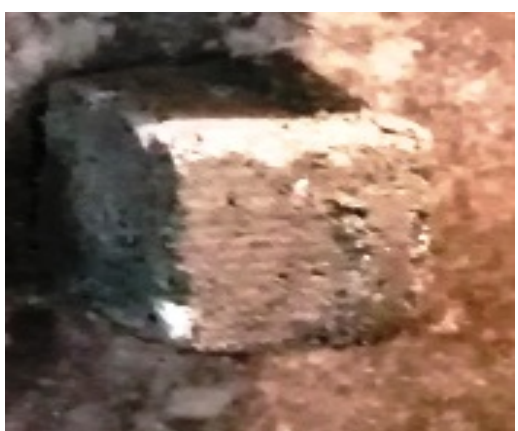

Figure 8: Eight Specimen 


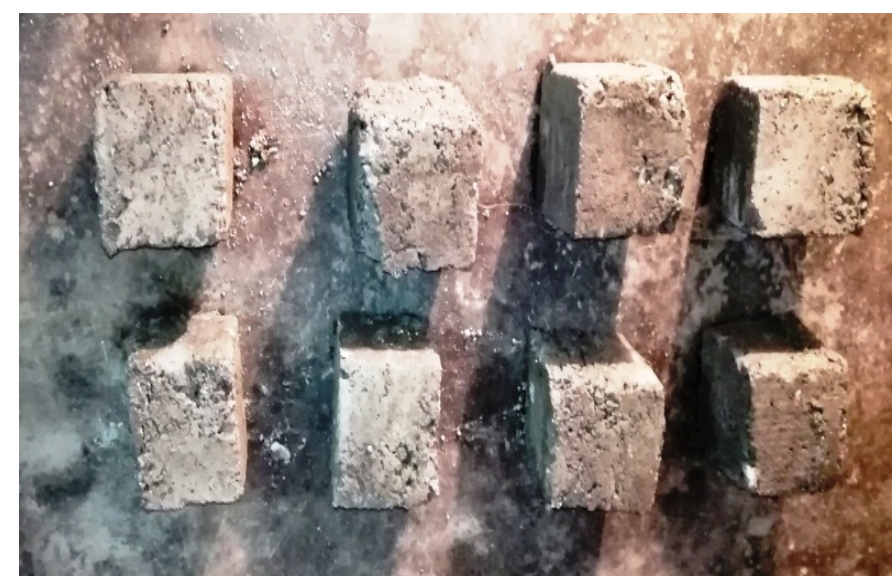

Figure 9: First to Eight Specimens

\section{RESULTS AND DISCUSSION}

Table 1: Properties of Cement

\begin{tabular}{|c|c|c|}
\hline Sr. No. & Property & Magnitudes \\
\hline 1 & Normal Consistency & $40 \%$ \\
\hline 2 & Initial Setting time & 60 minutes \\
\hline 3 & Specific Gravity & 3.15 \\
\hline 4 & Fineness of cement & $5 \%$ \\
\hline
\end{tabular}

Table 2: Properties of Fine Aggregate

\begin{tabular}{|c|c|c|}
\hline Sr. No. & Property & Magnitudes \\
\hline 1 & Specific Gravity & 2.66 \\
\hline 2 & Fineness modulus & 2.56 \\
\hline
\end{tabular}

Table 3: Properties of Silica Fume

\begin{tabular}{|c|c|c|}
\hline Sr. No. & Property & Magnitudes \\
\hline 1 & Specific Gravity & 2.2 \\
\hline 2 & Bulk Density & $576,\left(\mathrm{Kg} / \mathrm{m}^{3}\right)$ \\
\hline 3 & Size, (Micron) & 0.1 \\
\hline 4 & Surface Area, $\left(\mathrm{m}^{2} / \mathrm{kg}\right)$ & 20,000 \\
\hline 5 & $\mathrm{Si02}$ & $90 \%-96 \%$ \\
\hline 6 & $\mathrm{Al} 203$ & $0.5 \%-0.8 \%$ \\
\hline
\end{tabular}

Table 4: Mix

\begin{tabular}{|c|c|c|}
\hline Sr. No. & Material & Quantity in $\mathrm{Kg} / \mathrm{m}^{3}$ \\
\hline 1 & Cement (OPC) & 520 \\
\hline 2 & Fine Aggregate & 460.722 \\
\hline 3 & Coarse Aggregate & 1421.699 \\
\hline 4 & Water & 190.6 \\
\hline
\end{tabular}

Table 5: Results of Compressive Strength during 7 days

\begin{tabular}{|c|c|c|}
\hline Mix & $\begin{array}{c}\text { \% of Silica } \\
\text { Silica Fume } \\
\text { added }\end{array}$ & $\begin{array}{c}\text { Compressive } \\
\text { Strength }\left(\mathrm{Kg} / \mathrm{cm}^{2}\right)\end{array}$ \\
\hline M1 & 0 & 28.22 \\
\hline M2 & 5 & 32.66 \\
\hline M3 & 10 & 34.85 \\
\hline
\end{tabular}




\begin{tabular}{|l|l|l|}
\hline M4 & 15 & 36.58 \\
\hline M5 & 20 & 38.55 \\
\hline M6 & 25 & 41.66 \\
\hline M7 & 30 & 43.82 \\
\hline M8 & 35 & 42.91 \\
\hline
\end{tabular}

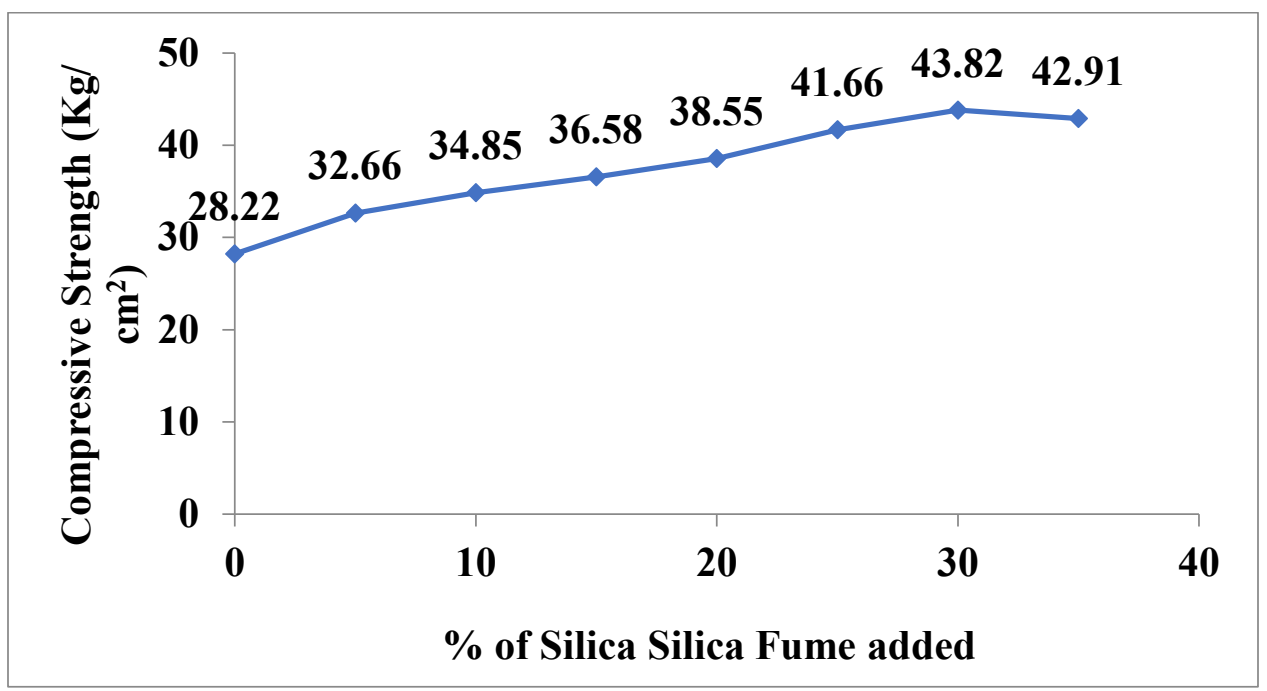

Figure 10: Results of Compressive Strength during 7 days

Table 6: Results of Compressive Strength during 28 days

\begin{tabular}{|c|c|c|}
\hline Mix & $\begin{array}{c}\text { \% of Silica } \\
\text { Silica Fume } \\
\text { added }\end{array}$ & $\begin{array}{c}\text { Compressive } \\
\text { Strength }\left(\mathrm{Kg} / \mathrm{cm}^{2}\right)\end{array}$ \\
\hline M1 & 0 & 29.55 \\
\hline M2 & 5 & 33.94 \\
\hline M3 & 10 & 35.88 \\
\hline M4 & 15 & 40.95 \\
\hline M5 & 20 & 44.98 \\
\hline M6 & 25 & 48.99 \\
\hline M7 & 30 & 50.22 \\
\hline M8 & 35 & 49.68 \\
\hline
\end{tabular}

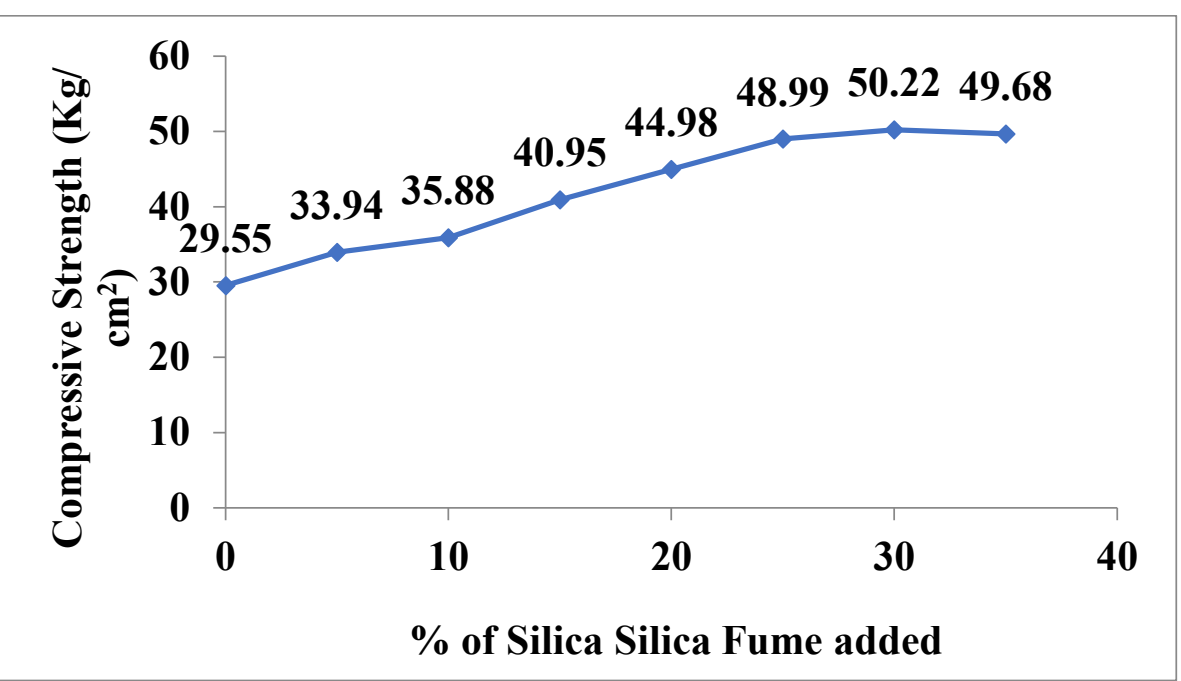

Figure 11: Results of Compressive Strength during 28 days 
Table 7: Results of Split tensile Strength during 7 days

\begin{tabular}{|c|c|c|}
\hline Mix & $\begin{array}{c}\text { \% of Silica } \\
\text { Silica Fume } \\
\text { added }\end{array}$ & Split tensile Strength $(\mathrm{Kg} / \mathrm{cm} 2)$ \\
\hline M1 & 0 & 4.22 \\
\hline M2 & 5 & 5.84 \\
\hline M3 & 10 & 6.21 \\
\hline M4 & 15 & 7.81 \\
\hline M5 & 20 & 8.94 \\
\hline M6 & 25 & 9.63 \\
\hline M7 & 30 & 10.21 \\
\hline M8 & 35 & 9.86 \\
\hline
\end{tabular}

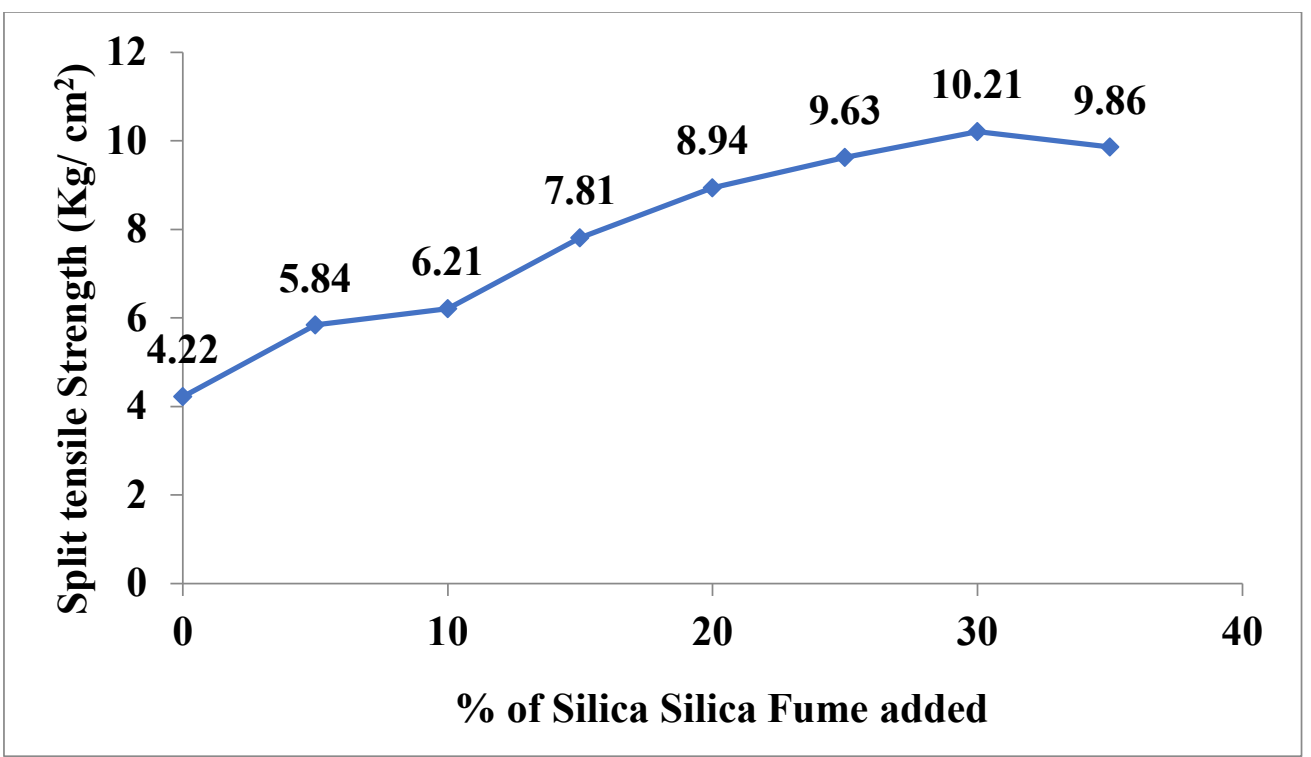

Figure 12: Results of Split tensile Strength during 7 days

Table 8: Results of Split tensile Strength during 28 days

\begin{tabular}{|c|c|c|}
\hline Mix & $\begin{array}{c}\text { \% of Silica } \\
\text { Silica Fume } \\
\text { added }\end{array}$ & $\begin{array}{c}\text { Split tensile } \\
\text { Strength }\left(\mathrm{Kg} / \mathrm{cm}^{2}\right)\end{array}$ \\
\hline M1 & 0 & 5.98 \\
\hline M2 & 5 & 6.88 \\
\hline M3 & 10 & 7.84 \\
\hline M4 & 15 & 8.94 \\
\hline M5 & 20 & 9.41 \\
\hline M6 & 25 & 10.64 \\
\hline M7 & 30 & 12.66 \\
\hline M8 & 35 & 11.56 \\
\hline
\end{tabular}


Kamar Elahi, and Prof. Harsh Gupta

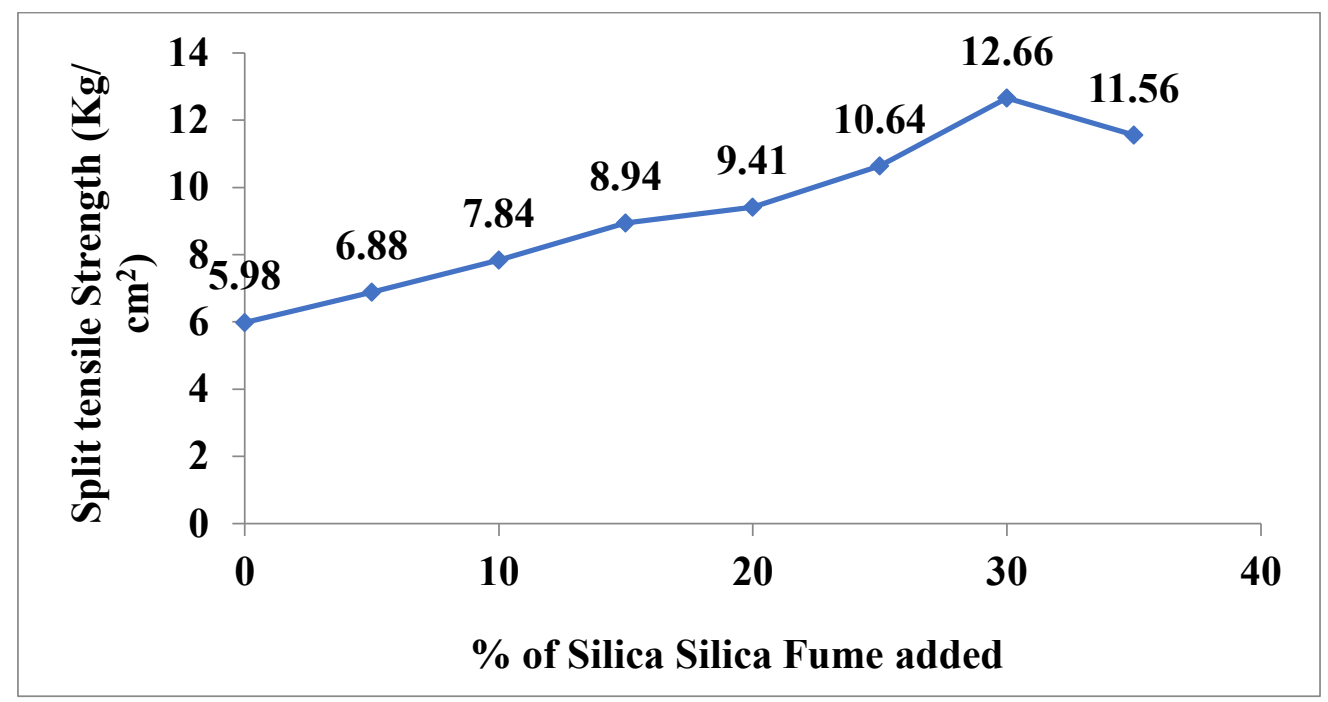

Figure 13: Results of Split tensile Strength during 28 days

\section{CONCLUSIONS}

We are tested the specimen size of $15 \mathrm{~cm}$ X $15 \mathrm{~cm}$ X $15 \mathrm{~cm}$ to observe the compressive strength $\left(\mathrm{Kg} / \mathrm{cm}^{2}\right)$ and split tensile strength $\left(\mathrm{Kg} / \mathrm{cm}^{2}\right)$. The various results are achieved with the help of \% of silica fume addition. These specimens are tested using compression testing machine after 7 days curing, after this optimum result are finding out using Mix (M7) which is shown Table 5, and Figure 10. Using of compression testing machine after 28 days curing, after this optimum result are finding out using of Mix (M7) which is shown Table 6 and Figure 11. These specimens are tested using compression testing machine after 7 days curing, after this optimum result are finding out using of Mix (M7) which is shown Table 7, and Figure 12. Using compression testing machine after 28 days curing, after this optimum result are finding out using of Mix (M7) which is shown Table 8, and Figure 13.

\section{SOURCES OF FUNDING}

This research received no specific grant from any funding agency in the public, commercial, or not-for-profit sectors.

\section{CONFLICT OF INTEREST}

The author have declared that no competing interests exist.

\section{ACKNOWLEDGMENT}

None.

\section{REFERENCES}

[1] Khayat, K. H. 1995. "Effects of anti-washout admixtures on fresh concrete properties." ACI Mater. J., pp 164171.

[2] Assaad, J. 2003. "Relationship between washout resistance and rheological properties of high-performance underwater concrete." ACI Mater. J., pp 185-193.

[3] Ballivy, G. 1996. "High-performance cement grout for underwater crack injection." Proc., 3rd CANMET/ACI Int. Conf. on Performance of Concrete in Marine Environment, V. M. Malhotra, ed., ACI, Farmington Hills, Mich., pp 138-162. 